\section{Transfusion related acute lung injury (TRALI)}

TAJANA ZAH • JASNA MESARIĆ • VIŠNJA MAJERIĆ-KOGLER

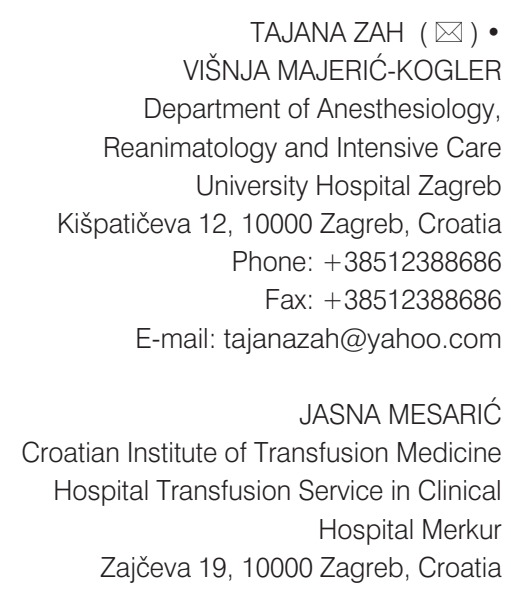

TAJANA ZAH $(\bowtie) \bullet$ VIŠNJA MAJERIĆ-KOGLER Department of Anesthesiology, Reanimatology and Intensive Care University Hospital Zagreb Kišpatičeva 12, 10000 Zagreb, Croatia

Phone: +38512388686

Fax: +38512388686

E-mail: tajanazah@yahoo.com

JASNA MESARIĆ

Croatian Institute of Transfusion Medicine Hospital Transfusion Service in Clinical Hospital Merkur

Zajčeva 19, 10000 Zagreb, Croatia

\begin{abstract}
Transfusion-related acute lung injury (TRALI) is a complication following transfusion of blood products and is potentially a life-threatening adverse event of transfusion. The first case of fatal pulmonary edema following transfusion was reported in the 1950s. In recent time, TRALI has developed from an almost unknown transfusion reaction to the most common cause of transfusion related major morbidities and fatalities. A clinical definition of TRALI was established in 2004, based on acute respiratory distress which has temporal association with transfusion of blood components. In 2008 a distinction between classic and delayed syndrome was proposed. However, pathophysiology of TRALI still remains controversial. A number of different models were proposed to explain the pathogenesis. The two, presently most accepted models, are not mutually exclusive. The first is the antibody mediated model and the second is the two-event model.

In this review article the definition of TRALI, patient predisposition, treatment, prevention and reporting guidelines are examined. The current knowledge on the topic TRALI is summarized.
\end{abstract}

Key words: transfusion, acute lung injury

\section{Introduction}

Transfusion-related acute lung injury (TRALI) is one of the most common causes of transfusion associated major morbidity and death. The results from many national haemovigillance programs have identified TRALI as a significant adverse event of transfusion, and the reported mortality rates vary from 5 to $25 \%$. (1-5) TRALI is a life-threatening adverse event of transfusion.

Investigators have shown that non-cardiogenic lung edema is also an important pulmonary complication of transfusion. Since Barnard's initial description in
1951, (6) non-cardiogenic lung edema related to transfusion has been widely reported using various names, including non-cardiogenic pulmonary edema, pulmonary hypersensitivity and severe allergic pulmonary edema. In 1983 Popovsky et al. $(7,8)$ coined the term "transfusion-related acute lung injury". The true incidence of TRALI is unknown but different estimates have been made. TRALI has been reported from all types of blood components including whole blood, red cells, aphaeresis platelets, whole blood platelets, fresh frozen plasma, cryoprecipitate, granulocytes, stem cell products and even IV immunoglobulin preparations. (9-13)

According to the literature, the inciden- ce is 1:5000 for blood components (8) with subsequent reports ranging from 1:432 for whole blood platelets to 1:557,000 for red cells. (14) The most implicated are plasma-rich components, such as plasma and aphaeresis platelets. $(14,15)$

TRALI is under- diagnosed and underreported as many clinicians are not familiar with the syndrome. This is equally true for severe cases of TRALI where transfusion may not be considered as a possible cause and may be regarded as acute respiratory distress syndrome (ARDS) but also for mild case where mechanical ventilation is not required. TRALI can be misdiagnosed as TACO (Transfusion Associated Circulatory 
Overload) as occurred in the French haemovigilance network. (2)

Patients who are at risk for developing TRALI are still not properly identified. Van Buren et al. first postulated that the clinical status of the patient played a significant role in the pathogenesis of TRALI. (16) According to some observations, patients in the induction phase of treatment for hematological malignancies and patients with cardiovascular disease who required bypass surgery have a higher risk for developing TRALI. (13)

\section{Clinical presentation}

TRALI is a clinical syndrome characterized by the acute onset of respiratory distress in a patient who received transfusion. It can present with a variety of symptoms and signs, most commonly worsening of respiratory function. Patients present with respiratory distress (dyspnea, tachypnea), hypoxia, hypotension (less commonly hypertension), fever, tachycardia and cyanosis, pulmonary edema (frothy pulmonary secretions), and bilateral fluffy infiltrates on chest radiograph. The majority of cases occur during or within 1 to $2 \mathrm{~h}$ of transfusion. (8)

\section{Definition}

The lack of a consensus definition of TRALI contributed to its under-recognition.

The TRALI definition of the European Haemovigilance Network (17) and Amendments by the TRALI Consensus Conference Committee in Toronto in $2004(17,18)$ is:

(a) Acute onset of acute lung injury (ALI)

(b) Hypoxemia $\left(\mathrm{PaO}_{2} / \mathrm{FiO}_{2}\right.$ (ratio of partial pressure of arterial $\mathrm{O}_{2}$ to the fraction of inspired $\mathrm{O}_{2}$ ) $\leq 300$ and must be adjusted downward with increasing altitude or $\mathrm{SpO}_{2} \leq 90 \%$ on room air or other clinical evidence)

(c) Bilateral lung infiltrates on frontal chest radiograph

(d) No evidence of left atrial hypertension (i.e., transfusion-associated circulatory overload) (e) Occurrence during or within $6 \mathrm{~h}$ after completion of transfusion

(f) No temporal relationship to an alternative risk factor for ALI

(g) New ALI and no other ALI risk factors present including aspiration, multiple trauma, pneumonia, cardiopulmonary bypass, burn injury, toxic inhalation, lung contusion, acute pancreatitis, drug overdose, near drowning, shock and sepsis

(h) If one or more ALI risk factors are present, possible TRALI should be diagnosed (in patients with an alternative ALI risk factor, TRALI is still possible)

Classic TRALI is therefore a clinical syndrome. Sometimes, a single unit of blood or blood product is implicated in causing the syndrome. Patients with classic TRALI should not have other risk factors for ALI and, thus, the transfusion is the only identifiable cause of the respiratory failure. (19)

Limitations to this definition are the exclusion of patients with preexisting ALI and cases with onset more than $6 \mathrm{~h}$ after transfusion. The modified criteria broadened the definition of hypoxia to include clinical evidence of hypoxia and created a category of "possible TRALI" to address cases in which patients have other risk factors for ALI such as sepsis, aspiration, near-drowning, disseminated intravascular coagulation, trauma, pneumonia, drug overdose, fracture, burns and cardiopulmonary bypass. (6) In recent years the term "delayed TRALI syndrome" emerged. (19) These patients usually have other risk factors for ARDS and do not meet the current definition for TRALI. $(17,18)$

Characteristics of the "classic TRALI syndrome" are: time of onset within 2 hours (usually up to 6 hours); rapid development; no other risk factors for ALI except transfusion; anti-neutrophil antibodies pathophysiology and onset after a single unit of blood product.

Characteristics of the "delayed TRALI syndrome" are: time of onset 6-72 hours after transfusion; slow development of clinical presentation; patients have other risk factors for ALI (i.e. sepsis, aspiration, near-drowning, disseminated intravascular coagulation, trauma, pneumonia, drug overdose, fracture, burns and cardiopulmonary bypass); two- step pathophysiology and common after massive transfusion (40-57\%). (19) This distinction is important, but undoubtedly there is some overlap between the two syndromes.

\section{Differential diagnosis}

The differential diagnosis includes TACO, cardiogenic edema, allergic and anaphylactic transfusion reactions, and transfusion of bacterially contaminated blood components, and classic ALI.

\section{Pathophysiology}

The exact mechanism of TRALI is still not fully understood. (19) At present we know that the common denominator is the end of the pathway which presents with increased pulmonary capillary permeability, and results in movement of plasma into the alveolar space causing pulmonary edema. Two possible theories have been proposed to explain the pathogenesis of TRALI. The first suggests an antibody mediated reaction after transfusion. The antibody-mediated model postulates that the reaction is secondary to passive transfusion of specific donor antibodies against recipient antigens (human leukocyte antigen (HLA) specific, mostly HLA-A2 or anti-granulocyte specific antibodies like human neutrophil antigen (HNA)-1a, $-1 b,-3 a$ or HLA 1) or infusion of donor antigens (leucocytes) in recipient who already has antibodies against them. This antibody-antigen interaction can cause complement activation, resulting in the pulmonary sequestration and activation of neutrophils, endothelial cell damage, and a capillary leak syndrome in the lungs manifesting as TRALI. (10,17-19) There is evidence that TRALI is more common in recipients of blood products from multiparous female donors who are more likely to possess anti-HLA antibodies and anti-neutrophil-specific antibodies. (20) However, in about $5-15 \%$ of cases, no antibody is identified in either the donor or recipient. The second is a two-event model. 
$(13,18-20)$ The first event (first hit) is the clinical condition of the patient resulting in pulmonary endothelial activation and polimorphonuclear (PMN) sequestration, and the second event is the transfusion of a biologic response modifier (including anti-granulocyte antibodies, lipids, and CD40 ligand) that activates these adherent PMNs resulting in endothelial damage, capillary leak, and TRALI. These two mechanisms of injury may not be mutually exclusive and a patient may develop TRALI through either one or both mechanisms.

\section{Prevention}

TRALI prevention is a complex issue, and comprehensive and effective preventive treatment steps are still unknown. $(19,21)$ Although it is probably impossible to prevent all cases of TRALI, there are certain measures that can be taken to at least prevent TRALI as much as possible.

Specific measures that could be considered in critical care practice include better compliance with evidence based triggers for transfusion, greater use of near patient testing to determine the need for transfusion, and minimizing blood loss due to sampling.

All TRALI reactions should be reported to the transfusion service. All associated donors are tested for HLA class I and II antibodies as well as HNA antibodies. As the transfused units are usually not available for testing, this involves recalling the donors. Associated donors may continue donating while being investigated but only plasma for fractionation is used. All implicated donors (i.e. donors who are cross match positive with the patient's leucocytes or donors who have the cognate antibody to HLA class I, II or neutrophil antigens of the patient) are permanently deferred from donation. The permanent deferral is considered consistent with the 2004 consensus conference statement (21) and is supported by two retrospective studies that showed that a TRALI-implicated donor may cause multiple transfusion reactions in different recipients.

\section{Management of the patient}

The management of both the classic and delayed TRALI syndrome is essentially supportive and includes gentle ventilation (lung protective ventilation).

\section{Conclusion}

Further work is required to define: 1) the effects of this life threatening syndrome; 2) patients who are at risk of TRALI and 3) a consensus international definition so that clinicians may properly diagnose TRALI

\section{REFERENCES}

1. Serious Hazards of Transfusion (SHOT): Additional Cumulative Data 1996-2003. Available from: http://www. shotuk.org

2. Renaudier PC, Vo Mai MP, Azanowsky JM, Breton P, Cheze S, Girard A, et al. Epidemiology of transfusion-related acute lung injury in Gifit, the French haemovigillance database. A study of the French hemovigilance network. Transfusion

2004;44:23-7.

3. Graul A, Heiden M, Gräf K, Keller-Stanislawski B. Hämovigilanz in Deutschland - Berichte an das Paul-Ehrlich-Institut über Verdachtsfälle von Transfusionsreaktionen im Beobachtungszeitraum Januar 1995 bis Dezember 2002.Transfus Med Hemother 2003;30:232-8

4. Joergensen J, Taaning E. DART - a volunteer system of haemovigillance in Denmark. Transfusion Alternatives. Transfus Med 2003;5:260-4.

5. Robillard P, Nawej KI, Chapdeleine A, Poole J, Garneau N. Adverse transfusion reactions with respiratory signs and symptoms including TRALI in the Quebec Haemovigillance system: 2000-2003.Transfusion 2004;44:23-9.

6. Barnard RD. Indiscriminate transfusion: a critique of case reports illustrating hypersensitivity reactions NY State. J Med 1951;51:2399402.

7. Popovsky MA, Abel MP, Moore SB. Transfusion-related acute lung injury associated with passive transfer of antileukocyte antibodies. Am Rev Respir Dis 1983;128:185-9.

8. Popovsky MA, Moore SB. Diagnostic and pathogenetic considerations in transfusion- related acute lung injury. Transfusion 1985;25(6):573-7.

9. Rizk A, Gorson KC, Kenney L, Weinstein R. Transfusion-related acute lung injury after the infusion of IVIG. Transfusion 2001;41:264-8

10. Toy P, Popovsky MA. Transfusion-related acute lung injury: Definition and review. Crit Care Med 2005;33:721-6.

11. Silliman CC, Paterson AJ, Dickey WO, Stroneck DF, Popovsky MA, Caldwell SA, et al. The association of biologically active lipids with the development of transfusion-related acute lung injury: A retrospective study. Transfusion 1997;37:719-26.

12. Wallis JP, Lubenko A, Wells AW, Chapman CE. Single hospital experience of TRALI. Transfusion 2003; 43:1053-9.

13. Silliman CC, Boskkov L, Mehdizadehkashi, Elzi DJ, Dickey WO, Podlosky L, et al. Transfusion related acute lung injury: epidemiology and a prospective analysis of etiologic factors. Blood 2003;101:454-62.

14. Goldman M, Webert KE, Arnold DM, Freedman J, Hannon J, Blajchman MA. Proceedings of a consensus conference: towards and understanding of TRALI. Transfus Med Rev 2005;19:2-31.

15. Jawa RS, Anillo S, Kulaylat MN. Transfusion-Related Acute Lung Injury. J Int Care Med 2008;23(2):109-21 
16. Van Buren NL, Stroncek DF, Clay ME, McCullough J, Dalmasso AP. Transfusion-related acute lung injury caused by an NB2 granulocytespecific antibody in a patient with thrombotic thrombocytopenic purpura. Transfusion 1990;30(1):42-5

17. European Haemovigillance Network (EHN): Definition of adverse transfusion events. Available from: http://www.ehn-org.net

18. Kleinmann S, Caulfield T, Chan P, Davenport R, McFarland J, McPhedran S, et al. Towards an understanding of transfusion - related acute lung injury: statement of a consensus panel. Transfusion 2004;44:1774-89.

19. Marik PE, Corwin HL. Acute lung injury following blood transfusion: Expanding the definition. Crit Care Med 2008;36:3080-4.

20. Bux J, Sachs UJH. The pathogenesis of transfusion-related acute lung injury (TRALI). Brit J Haemath 2007;136:788-99.

21. Mair DC, Hirschler N, Eastlund T. Blood donor and component management strategies to prevent transfusion-related acute lung injury( TRALI). Crit Care Med 2006;34:137-43. 